\title{
Development and Characterization of Novel Electrospun Meshes for Hernia Repair
}

\author{
Jason Chakroff, Doug Kayuha, Michaela Henderson, and Jed Johnson* \\ Nanofiber Solutions, Inc. Columbus, $\mathrm{OH}$, USA
}

Received: March 13, 2015; Accepted: April 30, 2015; Published: May 05, 2015

*Corresponding author: Jed Johnson, Nanofiber Solutions, 1275 Kinnear Road, Columbus, Ohio 43212, USA, Tel: +614-453-5877; Fax: +614-487-3704;

E-mail:Jed.Johnson@nanofibersolutions.com

\begin{abstract}
The use of surgical mesh for hernia repair has become widespread, as have the types of surgical mesh available on the commercial market. Biologic meshes are the most recent addition to the list of materials available for use in hernia repair, but the mechanical properties of these meshes are not always adequate for hernia repair applications. Electrospun polymer nanofibers have generated significant interest as a cell scaffolding material, but the literature characterizing these meshes for hernia repair applications is limited. In this work, novel electrospun nanofiber meshes made of PCL, PDO, PLGA1090, PLGA8218, PLLA, PU, and PET were successfully manufactured and characterized using standardized tests for hernia meshes from ISO and ASTM, including ball burst strength, suture retention strength, trouser tear strength, radial stiffness, and ultimate tensile strength in order to set a universal foundation that others can use in the future development of medical devices utilizing electrospun meshes. Surprisingly, the bioresorbable polymers outperformed the nonresorbable polymers in the mechanical tests. PCL, PLGA8218, and PLLA meshes had sufficient mechanical properties for use in hernia repair applications, but the PLLA mesh emerged as a favorable prospect for further development as a surgical mesh with an ultimate tensile strength of $35.85 \pm 2.45 \mathrm{~N} / \mathrm{cm}$, a suture retention strength of $17.3 \pm 4.3 \mathrm{~N}$ and a ball burst strength of $92.32 \pm 25.38 \mathrm{~N} / \mathrm{cm}$. Future work to characterize the in vivo response of the PLLA mesh will be needed to facilitate the translation of this electrospun hernia mesh to the clinic.
\end{abstract}

Keywords: Nanofibers; Hernia repair; Surgical mesh; Electrospinning; Tissue engineering

\section{Introduction}

Synthetic polypropylene mesh was first used for hernia repair in 1958 [1,2]. Surgical mesh was a significant improvement over suture-based methods due to the ability of the mesh to close defects and support the surrounding abdominal wall tissue [3]. As a result, the use of surgical mesh for hernia repair has since become extensive, as have the types of surgical mesh available on the commercial market. In addition to permanent polypropylene and polyethylene meshes, there are a plethora of resorbable meshes tailored to break down in vivo as the hernia site heals [4]. Meshes are often paired with visceral-side barrier layers to form composite materials that reduce the risk of adhesion to internal organs [5].

Biologic meshes are the most recent addition to the list of materials available for use in hernia repair. They are typically made of decellularized dermal matrix tissue derived from either human cadaveric or porcine sources [6]. These materials facilitate regenerative tissue formation and native collagen deposition prior to resorption and are often used in contaminated wound sites. Their mechanical properties, however, are not ideal for surgical mesh applications and it can be hard to predict how quickly the mesh will resorb in vivo leading to revision surgeries. Decellularized matrix materials also tend to undergo plastic deformation over time as a result of stress caused by intraabdominal pressure, which can lead to bulging of the abdominal wall and recurrent hernia. Biologic materials alone are therefore insufficient for bridging fascial defects [7].

Electrospun polymer nanofibers have generated significant interest as a cell scaffolding material. These fibers have diameters in the sub-micron range and replicate the morphology of the extracellular matrix found throughout the body. The high surface area to volume ratio of nanofiber mesh promotes cell adhesion and proliferation, while the high porosity promotes nutrient and gas exchange [8]. Nanofibers can be electrospun using biocompatible polymers with a wide range of in vivo resorption periods and minimal inflammatory reactions. Furthermore, through polymer selection and manipulation of electrospinning parameters, it is possible to fine-tune the mechanical properties of electrospun scaffolds $[9,10]$. Fibers can also be spun such that they are predominately oriented in the same direction, which can result in anisotropic mechanical properties and control of cell orientation $[11,12]$.

A typical electrospinning setup consists of a high voltage power source connected to the tip of a syringe with a metal needle. The syringe is filled with a viscous polymer solution and placed in a pump set to dispense the solution at a constant rate. As the solution flows from the tip of the syringe, it is charged by the high voltage power source, which exerts electrostatic forces on the solution at the tip of the needle, and causes it to form a Taylor cone. A jet of solution ejects from the Taylor cone and destabilizes in mid-air. As the solvent evaporates, solid 
nanofibers form and are deposited on to a grounded collector. The initial costs of equipment can be high, however the overall electrospinning process is quick and inexpensive.

Electrospun nanofiber scaffolds have the potential to provide a cheap hernia mesh with customized mechanical properties, degradation rates, drug release capabilities, and an excellent capacity to support native tissue regeneration, but there is a dearth of knowledge available on electrospun hernia meshes [13]. The goal of this study was to characterize the mechanical properties of a series of novel electrospun meshes made from the bioresorbable polymers: Polycaprolactone (PCL), Polydioxanone (PDO), Polylactide-Co-Glycolide (PLGA1090), Polylactide-CoGlycolide (PLGA8218), and Poly-L-Lactide (PLLA), and the non-resorbable polymers Polyurethane (PU) and Polyethylene Terephthalate (PET) in order to select a suitable candidate for use in surgical mesh applications.

\section{Materials and Methods}

Eight polymer compositions were electrospun into nonwoven mats using a custom designed electrospinning setup. All electrospinning was performed with Hexafluoroisopropanol (HFIP) (Sigma Aldrich) used without modification. Solutions were prepared by adding the appropriate amount of polymer and solvent into an Erlenmeyer flask, and stirring continuously until the polymer was completely dissolved. The polymer solutions consisted of 5wt\% PCL (Sigma Aldrich), 7wt\% PDO (Evonik), 8wt\% PLGA1090 (Purac), 6wt\% PLGA8218 (Purac), 8wt\% PLLA (Goodfellow), 3wt\% PU (DSM), and 8wt\% PET (Auriga). These concentrations allowed the solutions to be spun at relatively high flow rates while maintaining uniform fiber morphology without beads. Meshes of each material were spun on to a slow-rotating cylindrical collector with a diameter of $8 \mathrm{~cm}$ and length of $17 \mathrm{~cm}$. A negative voltage was applied to the collector using an alternate power source. Meshes were spun to a thickness of approximately $250 \mu \mathrm{m}$. See Table I for detailed spin parameters.

Mechanical tests were performed using a Universal Testing Machine (MTS Systems Corporation) fitted with a $50 \mathrm{lb}$. load cell and set to a displacement rate of $50 \mathrm{~mm} / \mathrm{min}$. Data was analyzed using Microsoft Excel and reported as mean \pm standard deviation. The tests performed included Ball Burst Strength (BBS), Radial Stiffness (RS), Trouser Tear Strength (TTS), Suture Retention Strength (SRS), and Ultimate Tensile Strength (UTS). Mesh thickness was measured and reported, as well as Mesh Density. All tests were performed according to ASTM standards except suture retention strength, which followed ISO standards. Tests were performed using five samples of each mesh material.

\section{Scanning electron microscopy}

Electrospun nanofiber meshes were sputter coated with a 5 $\mathrm{nm}$ thick layer of gold for observation in an FEI Nova 400 scanning electron microscope (SEM). Representative SEM images of each material were taken. One image of each material was analyzed using FibraQuant software from Nanoscaffold Technologies. Approximately 500 fiber diameter measurements were made per image. Average fiber diameters were calculated and recorded.

\section{Thickness measurements}

The thickness of every mesh test sample was measured prior to testing. A digital caliper (VWR) with a readability of 0.00 mm was zeroed on two $3 " \times 1 " \times 1 \mathrm{~mm}$ glass microscope slides (VWR). Samples were placed between the glass slides, and the thicknesses were measured following ASTM standard D6988-13. Three measurements were taken, one on the right, center, and left of each sample. The median of these three measurements was recorded in millimeters as the thickness of the sample. Actual sample thicknesses were then used to convert test results from Newtons, to Newtons per millimeter thickness, and then finally to Newtons assuming a mesh thickness of $1 \mathrm{~mm}$. This normalized the thickness of every mesh and allowed small differences in the thicknesses of the meshes to be resolved so that they would not skew mechanical data and allowed us to compare our nanofiber meshes to commercially available hernia meshes. Samples of various thicknesses were tested to validate this assumption.

\section{Density measurements}

Five samples of each mesh composition were cut into 2" $\times$ 2 " sheets. The length and width of each sample was recorded in mm following ASTM standard D3776/D3776-09a. The thickness of each sample was then determined as described above and the average sample density was calculated in Grams per Square Meter (GSM) assuming a sheet thickness of $1 \mathrm{~mm}$.

\section{Ball burst strength}

Five 3" $\times 3$ " sheets of each mesh composition were clamped between two metal rings and tightly secured for testing following ASTM standard D3787-07. During the testing of PU, PLGA8218, and PLGA1090 meshes, the metal rings were lined with sandpaper to prevent the meshes from slipping during the ball burst test. A metal plunger with a $2.5 \mathrm{~cm}$ diameter ball on the end was then pressed into the sheet until the ball burst through it. The peak load from each sample was averaged and recorded in Newtons per centimeter $(\mathrm{N} / \mathrm{cm})$ assuming $1 \mathrm{~mm}$ thick mesh.

\section{Radial stiffness}

Five samples of each mesh composition were cut into 2 " $\times$ 2 " sheets. Each sample was centered on a metal platform with a 1.5" diameter hole in the center following ASTM standard D403208. A 1" diameter plunger was then positioned $1 / 8$ " above the platform and pressed into the mesh until the plunger and mesh travelled 1.5" through the hole. The peak load from each sample was averaged and recorded in Newtons (N) assuming a $1 \mathrm{~mm}$ thick sheet.

\section{Trouser tear strength}

Five samples of each mesh composition were cut into 3 " $\times$ 1 " sheets, with a 2" long incision cut down the middle of the 1 " side, creating two tabs each with 0.5 " width. The two tabs were then secured in two vertically aligned grips, with one tab inserted into the upper grip and one inserted into the lower grip following ASTM standard D1938-08. The clamps then pulled the sample apart until the sample was torn in half. The peak load from each 
sample was averaged and recorded in Newtons (N) assuming a 1 mm thick sheet.

\section{Suture retention strength}

Five $1 \times 2 \mathrm{~cm}$ sheets of each mesh composition was cut. A 2-0 nylon suture was passed through the mesh $2 \mathrm{~mm}$ from the $1 \mathrm{~cm}$ long edge of each sample and tied to form a loop. The knotted end of the suture was secured in the upper clamp and the mesh test sample was secured in the bottom clamp. The suture was then pulled until it tore through the sample following ISO 7198. The peak load from each sample was averaged and recorded in Newtons (N) assuming a $1 \mathrm{~mm}$ thick sheet.

\section{Uniaxial tensile testing}

Five tensile dogbones were cut from sheets of each mesh composition according to ASTM D638 Type V. The ends of each dogbone sample were clamped to opposite arms of the testing machine and pulled apart until failure. The peak load from each sample was averaged and recorded in Newtons per centimeter $(\mathrm{N} / \mathrm{cm})$ assuming a $1 \mathrm{~mm}$ thick sheet. The elongation to failure of each sample was also recorded. Elastic modulus of each sample was determined from the slope of the linear regime of the resulting stress-strain curve. The average elastic modulus of each sample was recorded in MPa.

\section{Statistics}

Single sample t-tests were performed comparing the average test results of every material to generally accepted threshold values in the published literature. The threshold values were BBS: $50 \mathrm{~N} / \mathrm{cm}$, SRS: $20 \mathrm{~N}$, TTS: $20 \mathrm{~N}$, UTS: $16 \mathrm{~N} / \mathrm{cm}$ [1,4,14]. There is no generally accepted threshold value for radial stiffness. Single sample t-tests were also performed comparing the results of each material to published mechanical values of MotifMESHTM (Proxy Biomedical), an FDA-approved commercial surgical mesh. The test values of MotifMESHTM were BBS: $43.56 \mathrm{~N} / \mathrm{cm}$, SRS: $27.7 \mathrm{~N}$, TTS: $15.1 \mathrm{~N}$, RS: $2.8 \mathrm{~N}$, UTS: $105.3 \mathrm{~N} / \mathrm{cm}$ [15]. T critical values were calculated using the following equation:

$$
t=\frac{\bar{x}-\mu_{0}}{s / \sqrt{n}}
$$

In which $\mathrm{t}$ is the $\mathrm{t}$-critical value, $\mathrm{x}$ is the sample mean, $\mu_{0}$ is the control value, $s$ is the standard deviation of the sample group, and $n$ is the number of samples in the group. Degrees of Freedom were equal to $n-1$. P values were calculated using Microsoft
Excel for a right tailed t-distribution with $\alpha=0.05$.

\section{Results}

The mesh characterization data is summarized in Table II.

\section{Scanning electron microscopy}

Low (100 $\mu \mathrm{m}$ scale bar) and high $(10 \mu \mathrm{m}$ scale bar $)$ magnification SEM images of each composition are shown in Figures 1 and Figure 2. Fiber diameter data is summarized in Table II. All meshes were composed of solid, uniform fibers. No signs of beads were observed, nor were there any indications that the fibers were wet or bonded to one another. The average fiber diameters of each material were PLGA1090: $3.28 \pm 0.57 \mu \mathrm{m}$, PET: $2.47 \pm 0.69 \mu \mathrm{m}$, PLLA: $1.48 \pm 0.67 \mu \mathrm{m}$, PLGA8218: $1.28 \pm$ $0.38 \mu \mathrm{m}$, PU: $0.89 \pm 0.33 \mu \mathrm{m}$, PDO: $0.86 \pm 0.42 \mu \mathrm{m}$, and PCL: 0.64 $\pm 0.32 \mu \mathrm{m}$.

\section{Density measurements}

Mesh density values are displayed in Figure 3 and Table II. PCL had the lowest mesh density of 157 GSM, while PLGA8218 had the highest mesh density of 285 GSM. The rest of the mesh densities were PDO: 210 GSM, PLGA1090: 263 GSM, PLLA: 222 GSM, PU: 256 GSM, PET: 194 GSM.

\section{Ball burst strength}

BBS values are presented in Figure 4 and Table II. PET mesh had a BBS of $29.2 \mathrm{~N} / \mathrm{cm}$, the lowest of all the mesh compositions evaluated. The strongest materials tested were the PLLA and PU meshes, which had BBS of $92.3 \mathrm{~N} / \mathrm{cm}$ and $91.3 \mathrm{~N} / \mathrm{cm}$, respectively. PET was the only mesh tested with a BBS significantly lower than the $50 \mathrm{~N} / \mathrm{cm}$ threshold. The BBS of PCL and PLGA8218 were not significantly different than the $50 \mathrm{~N} / \mathrm{cm}$ threshold. PDO, PLGA1090, PLLA, and PU all had BBS significantly higher than the $50 \mathrm{~N} / \mathrm{cm}$ threshold. All meshes tested had significantly higher BBS than MotifMESHTM except PET, which had a significantly lower BBS.

\section{Radial stiffness}

Radial Stiffness values are portrayed in Figure 5 and Table II. PLGA1090 and PLGA8218 meshes had the highest RS values of $5.0 \mathrm{~N}$ and $4.0 \mathrm{~N}$, respectively. PCL and PDO meshes had the lowest RS values of $0.53 \mathrm{~N}$ and $0.57 \mathrm{~N}$, respectively. PCL, PDO, PU, and PET all had significantly lower RS than MotifMESHTM. The RS of PLGA8218 and PLLA were not significantly different from

Table 1: Summary of electrospinning parameters.

\begin{tabular}{|l|l|l|l|c|c|}
\hline Polymer & Distance $\mathbf{( c m )}$ & Flow Rate (ml/hr) & Voltage (kV) & Needle Gauge & \multicolumn{1}{|c|}{ Needle Inner $\emptyset$ (mm) } \\
\hline PCL & 20 & 5 & $+12 /-4$ & $+12 /-5$ & 21 \\
\hline PDO & 20 & 5 & $+14 /-2$ & 21 \\
\hline PLGA1090 & 20 & 5 & $+14 /-3$ & 21 \\
\hline PLGA8218 & 5 & 5 & $+14 /-3$ & 21 \\
\hline PLLA & 5 & $+12 /-3$ & 0.61 \\
\hline PU & 20 & 5 & $+12 /-3$ & 21 \\
\hline PET & 20 & 5 & 0.61 \\
\hline
\end{tabular}


Table 2: Summary of mesh characterization data.

\begin{tabular}{|c|c|c|c|c|c|c|c|c|c|c|}
\hline Polymer & $\begin{array}{l}\text { Mesh } \\
\text { Density } \\
\text { (GSM) }\end{array}$ & $\begin{array}{c}\text { Fiber Diam- } \\
\text { eter }(\mu \mathrm{m}) \\
\mathbf{n}=\mathbf{5 0 0}\end{array}$ & $\begin{array}{c}\text { Tensile } \\
\text { Strength } \\
(\mathrm{MPa}) \mathrm{n}=5\end{array}$ & $\begin{array}{c}\text { Tensile } \\
\text { Strength (N/ } \\
\mathrm{cm}) \mathrm{n}=5\end{array}$ & $\begin{array}{c}\% \\
\text { Elongation } \\
\mathbf{n}=5\end{array}$ & $\begin{array}{c}\text { Elastic } \\
\text { Modulus } \\
(\mathrm{MPa}) \mathrm{n}=5\end{array}$ & $\begin{array}{c}\text { Suture } \\
\text { Retention } \\
\text { Strength } \\
\text { (N) } n=5\end{array}$ & $\begin{array}{c}\text { Radial Stiff- } \\
\text { ness } \\
\text { (N) } n=5\end{array}$ & $\begin{array}{l}\text { Ball Burst } \\
\text { Strength (N/ } \\
\text { cm) } n=5\end{array}$ & $\begin{array}{l}\text { Trouser } \\
\text { Tear } \\
\text { Strength } \\
\text { (N) } n=5\end{array}$ \\
\hline PCL & $57+12$ & $0.64 \pm 0.32$ & $2.00 \pm 0.51$ & $20.06 \pm 5.13$ & $327 \pm 32$ & $2.07 \pm 0.57$ & $19.8 \pm 5.7$ & $0.53 \pm 0.08$ & $50.60 \pm 6.13$ & $31.9 \pm 9.3$ \\
\hline PDO & $10 \pm 10$ & $0.86 \pm 0.42$ & $3.76 \pm 0.49$ & $37.74 \pm 4.87$ & $575 \pm 128$ & $6.7 \pm 1.5$ & $10.1 \pm 2.5$ & $0.57 \pm 0.13$ & $60.03=$ & $12.3 \pm 1.0$ \\
\hline PLGA1090 & $263 \pm 29$ & $3.28 \pm 0.57$ & $6.47 \pm 0.41$ & $64.87 \pm 1.82$ & $399 \pm 34$ & $83.5 \pm 8.6$ & $13.2 \pm 2.1$ & $5.0 \pm 1.2$ & $79.98 \pm 15.07$ & $12.8 \pm 0.6$ \\
\hline PLGA8218 & $285 \pm 18$ & $1.28 \pm 0.38$ & $6.60 \pm 1.40$ & $65.7 \pm 13.8$ & $136 \pm 12$ & $103.3 \pm 4.6$ & $28.1 \pm 2.7$ & $4.0 \pm 1.7$ & $64.50 \pm 17.98$ & $23.9 \pm 4.8$ \\
\hline PLLA & $222 \pm 20$ & $1.48 \pm 0.67$ & $3.59 \pm 0.25$ & $35.85 \pm 2.45$ & $257 \pm 74$ & $49.5 \pm 4.1$ & $17.3 \pm 4.3$ & $2.53 \pm 0.53$ & $92.32 \pm 25.38$ & $25.9 \pm 8.6$ \\
\hline PU & $256 \pm 44$ & $0.89 \pm 0.33$ & $18.9 \pm 5.9$ & $189.6 \pm 59.1$ & $1107 \pm 100$ & $1.4 \pm 0.5$ & $17.5 \pm 2.7$ & $0.65 \pm 0.45$ & $91.32=$ & $16.6 \pm 9.0$ \\
\hline PET & $194 \pm 16$ & $2.47 \pm 0.69$ & $4.02 \pm 0.60$ & $40.25 \pm 5.94$ & $434 \pm 88$ & $14.1 \pm 2.7$ & $12.6 \pm 4.5$ & $0.88 \pm 0.16$ & $29.23 \pm 7.84$ & $6.0 \pm 0.6$ \\
\hline Threshold & $\mathrm{n} / \mathrm{a}$ & $\mathrm{n} / \mathrm{a}$ & & & $\mathrm{n} / \mathrm{a}$ & & 20 & $\mathrm{n} / \mathrm{a}$ & 50 & 20 \\
\hline MotifMESH & $\mathrm{n} / \mathrm{a}$ & $\mathrm{n} / \mathrm{a}$ & 70.23 & 105.3 & $\mathrm{n} / \mathrm{a}$ & $\mathrm{n} / \mathrm{a}$ & 27.7 & 2.8 & 43.56 & 15.1 \\
\hline
\end{tabular}

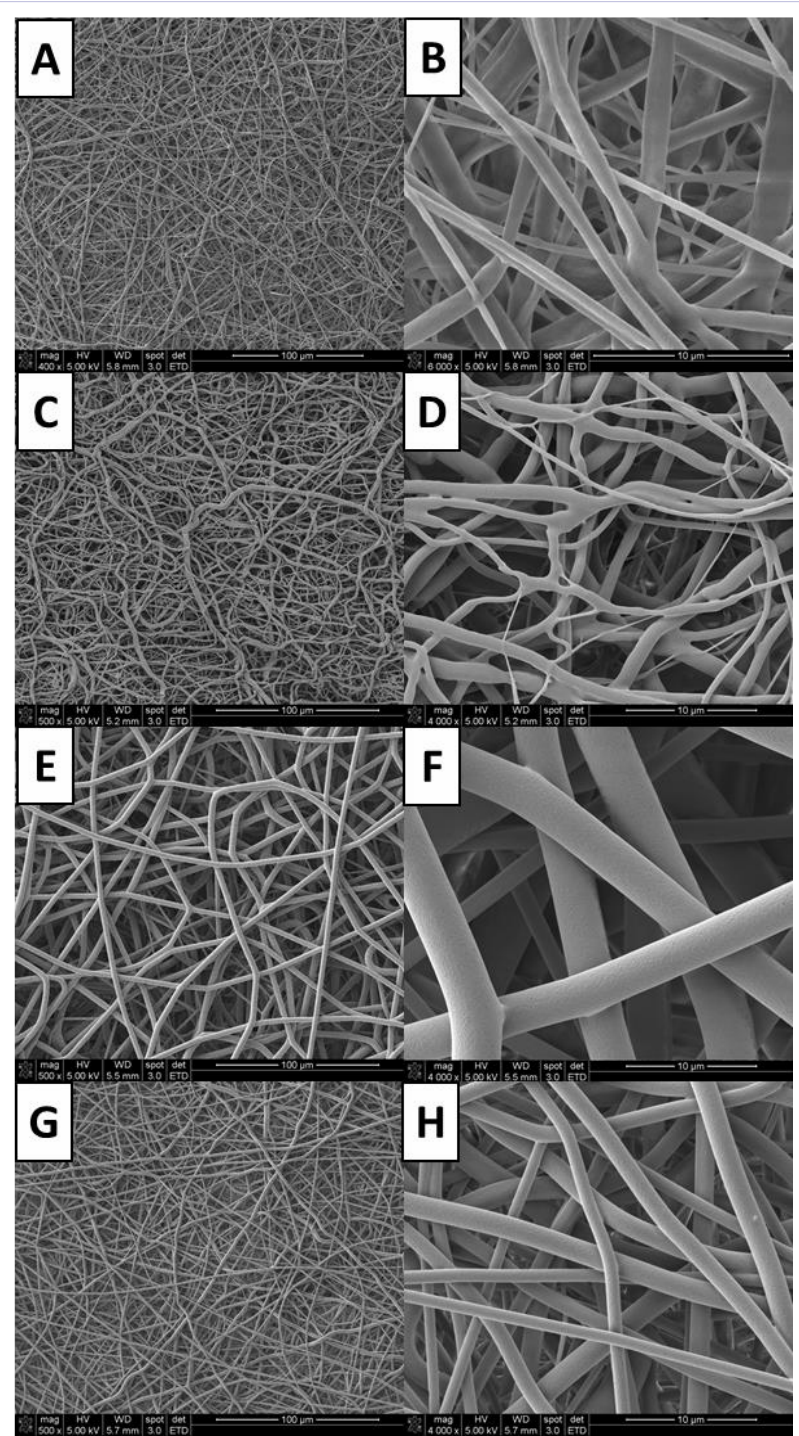

Figure 1: Panel of low magnification (left column, $100 \mu \mathrm{m}$ scale bar) and high magnification (right column, $10 \mu \mathrm{m}$ scale bar) SEM images of PCL (a,b), PDO (c,d), PLGA1090 (e,f), and PLGA8218 (g,h).
MotifMESHTM. PLGA1090 was the only mesh tested with a RS higher than MotifMESHTM.

\section{Trouser tear strength}

TTS data is displayed in Figure 6 and Table II. PET had the lowest TTS with a value of $6.0 \mathrm{~N}$, while the PCL mesh had the highest TTS of the polymer compositions tested with a value of 31.9 N. PDO, PLGA1090, and PET meshes had TTS values that

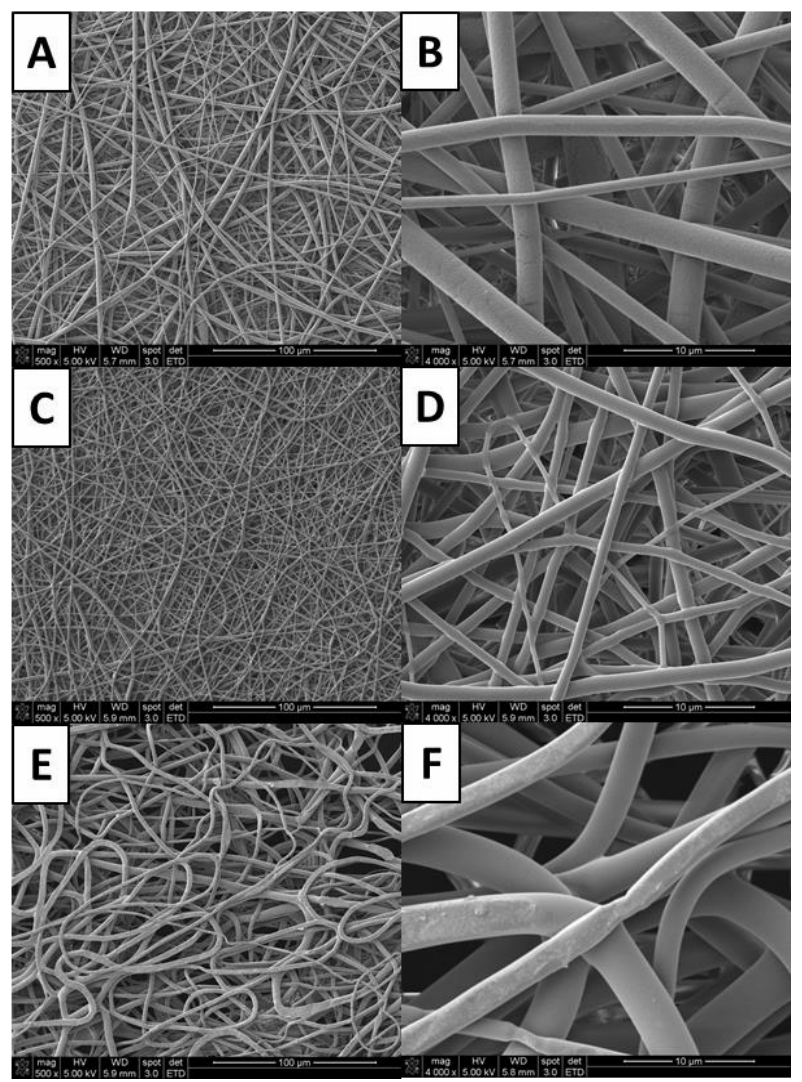

Figure 2: Panel of low magnification (left column, $100 \mu \mathrm{m}$ scale bar) and high magnification (right column, $10 \mu \mathrm{m}$ scale bar) SEM images of PLLA (a,b), PU (c,d), and PET (e,f). 

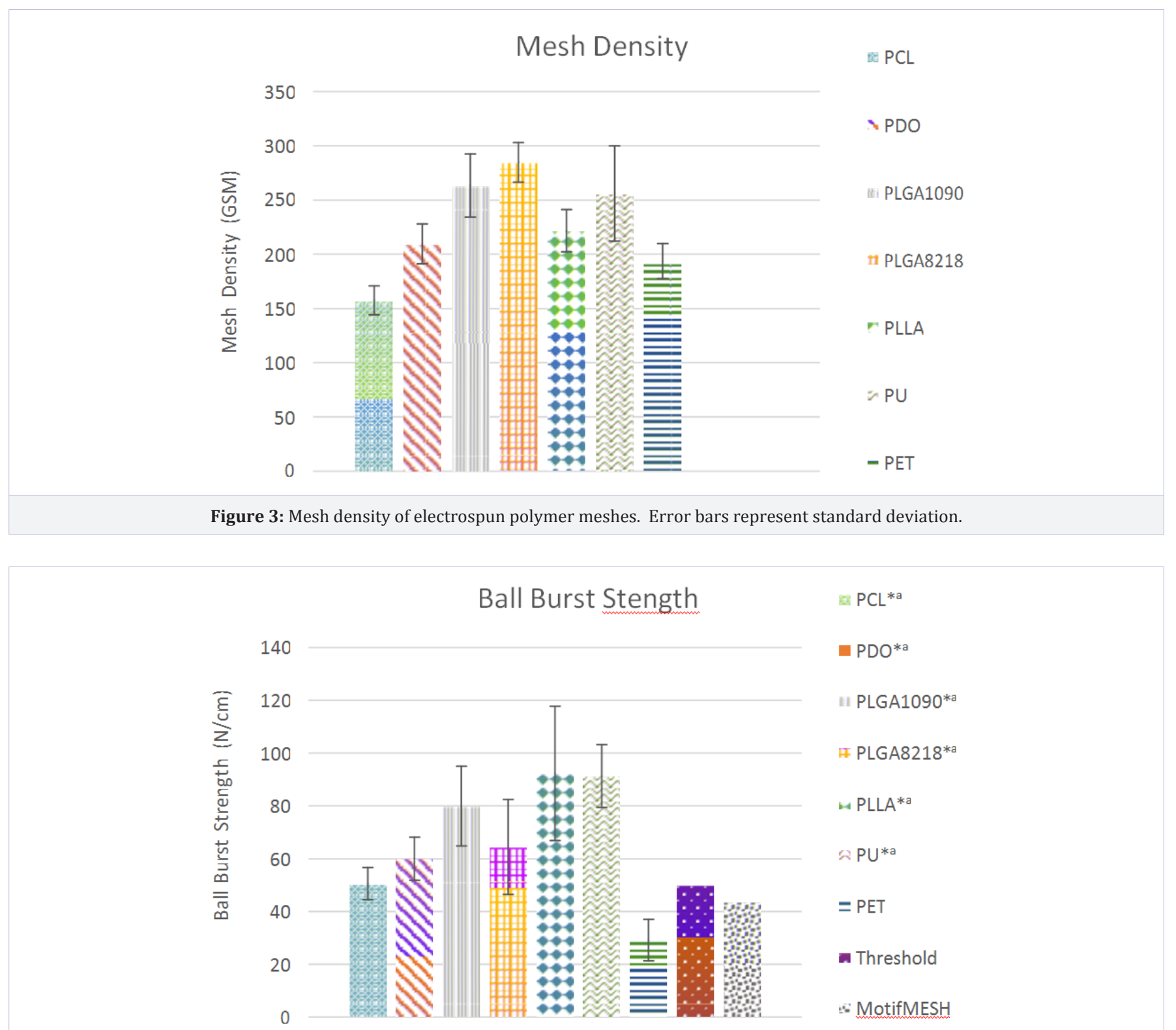

Figure 4: Ball Burst Strength values of electrospun polymer meshes, MotifMESHTM, and published threshold value. Error bars represent standard deviation when available.

* Material met or exceeded threshold value

a Material met or exceeded MotifMESHTM

were significantly lower than the $20 \mathrm{~N}$ threshold value. The TTS of PLGA8218, PLLA, and PU were not significantly different from the $20 \mathrm{~N}$ threshold values. The TTS of PCL was significantly higher than the $20 \mathrm{~N}$ threshold values. PDO, PLGA1090, and PET all had TTS values significantly lower than that of MotifMESHTM. The TTS of PU was not significantly different than MotifMESHTM. PCL, PLGA8218, and PLLA had TTS values higher than that of MotifMESHTM.

\section{Suture retention strength}

Suture retention strength values are displayed in Figure 7 and Table II. The PLGA8218 mesh had the highest SRS with a value of $28.1 \mathrm{~N}$. PDO had the lowest SRS of $10.1 \mathrm{~N}$. The SRS of PDO, PET, and PLGA1090 were all significantly lower than the $20 \mathrm{~N}$ threshold values. The SRS of PCL, PLLA, and PU were not significantly different from the $20 \mathrm{~N}$ threshold values. PLGA8218 was the only material with a SRS significantly higher than $20 \mathrm{~N}$. PLGA8218 was also the only mesh tested that was not significantly different than the MotifMESHTM control. All other meshes had a significantly lower SRS than MotifMESHTM.

\section{Uniaxial tensile testing}

Uniaxial tensile testing data is displayed in Table II and Ultimate Tensile Strength data is presented in Figure 8. PU mesh 


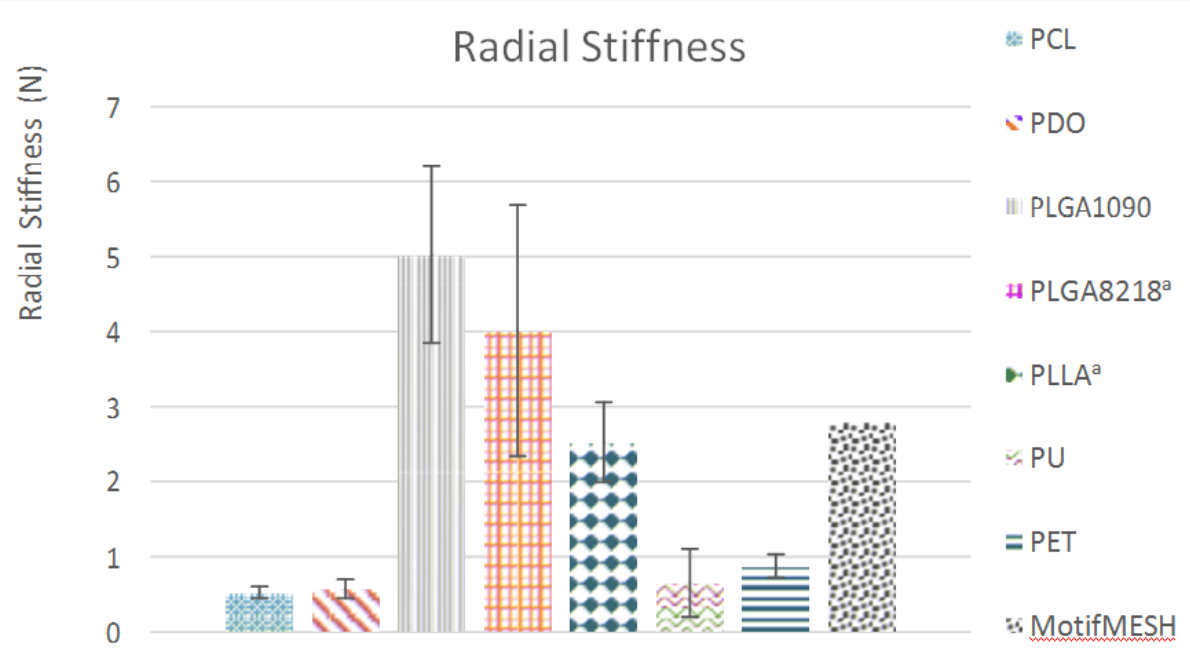

Figure 5: Radial stiffness of electrospun polymer meshes and MotifMESH ${ }^{\mathrm{TM}}$.

a Material stiffness was equivalent to MotifMESH ${ }^{\mathrm{TM}}$

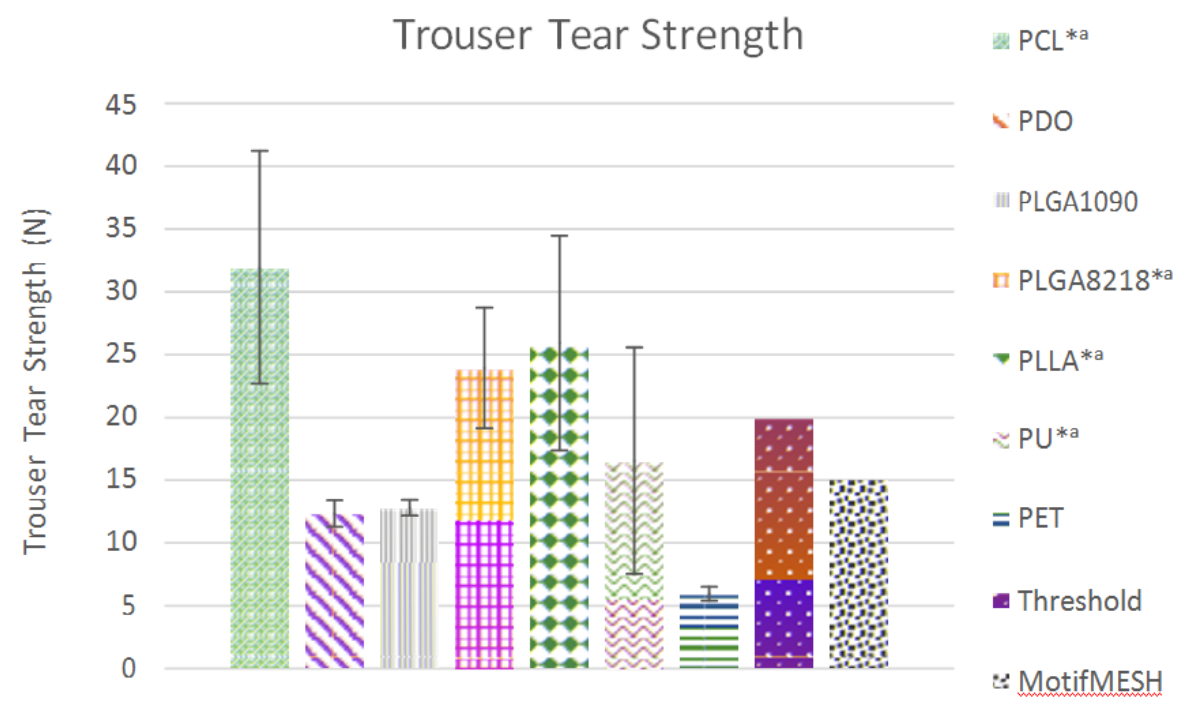

Figure 6: Trouser tear strength of electrospun polymer meshes, MotifMESH ${ }^{\mathrm{TM}}$, and published threshold value. Error bars represent standard deviation when available.

*Material met or exceeded threshold value

a Material met or exceeded MotifMESH ${ }^{\mathrm{TM}}$

had the highest UTS of $189.6 \mathrm{~N} / \mathrm{cm}$, while the PCL mesh had the lowest UTS of $20.1 \mathrm{~N} / \mathrm{cm}$. PU had the highest elongation to failure of $1,107 \%$ and PLGA8218 had the lowest elongation to failure at $136 \%$. PLGA8218 had the highest elastic modulus of $103.3 \mathrm{MPa}$ and PU had the lowest elastic modulus at $1.4 \mathrm{MPa}$. Every mesh tested had UTS significantly higher than the $16 \mathrm{~N} / \mathrm{cm}$ threshold value except PCL, which was not significantly different than the threshold value. Every mesh tested had significantly lower UTS than MotifMESHTM except PU, which had significantly higher UTS.

\section{Discussion}

This study was conducted in order to characterize the mechanical properties of nanofiber meshes made of various bioresorbable and non-resorbable polymers for hernia repair and act as a point of reference for the tissue engineering field in the future development of electrospun scaffolds. The ideal hernia mesh should be lightweight and flexible to reduce pain and foreign body sensation, as well as maintaining sufficient strength and durability to bridge fascial defects with minimal risk of recurrent hernia [1]. Through careful design, electrospun nanofiber meshes can balance these contradictory mechanical properties and provide a compromise between the mechanical stability of macrofiber meshes and the biocompatibility of biologically derived meshes.

In order to be considered sufficient for use in hernia repair applications, a mesh must have a minimum BBS of $50 \mathrm{~N} / \mathrm{cm}$, SRS 


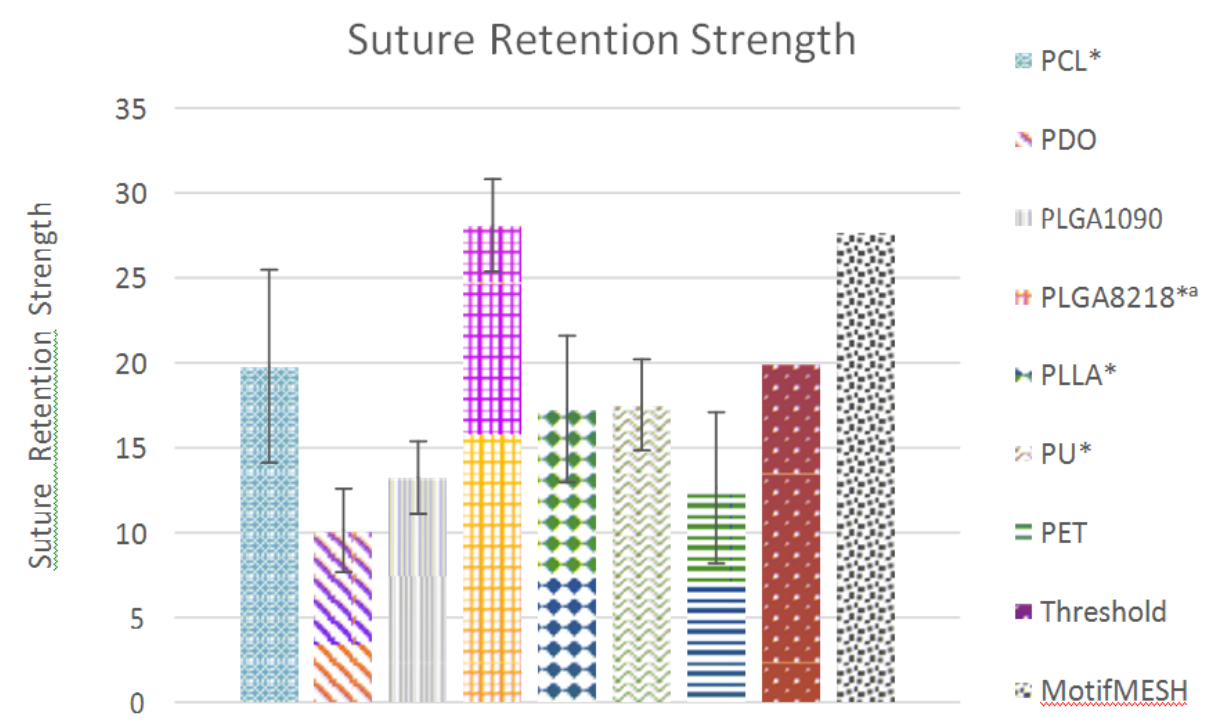

Figure 7: Suture retention strength of electrospun polymers, MotifMESH ${ }^{\mathrm{TM}}$, and published threshold value. Error bars represent standard deviation when available.

* Material met or exceeded threshold value

a Material met or exceeded MotifMESH ${ }^{\mathrm{TM}}$

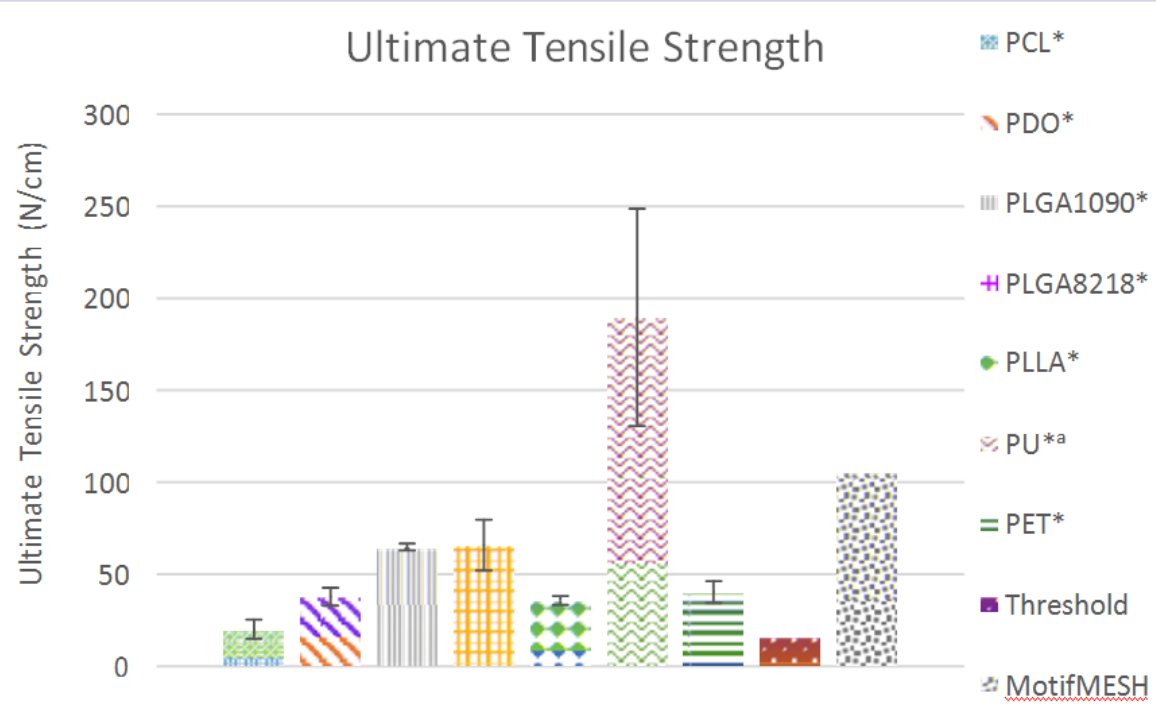

Figure 8: Ultimate tensile strength of electrospun polymer meshes, MotifMESH ${ }^{\mathrm{TM}}$, and published threshold value. Error bars represent standard deviation when available

* Material met or exceeded threshold value

a Material met or exceeded MotifMESH ${ }^{\mathrm{TM}}$

of $20 \mathrm{~N}$, TTS of $20 \mathrm{~N}$, and UTS of $16 \mathrm{~N} / \mathrm{cm}[1,4,14]$. Based on the statistical analyses, every mesh tested met or surpassed the 50 N/cm BBS threshold except PET. PCL, PLGA8218, PLLA, and PU met or surpassed the $20 \mathrm{~N}$ SRS threshold, as well as the $20 \mathrm{~N}$ TTS threshold. All of the meshes tested met or surpassed the UTS threshold value of $16 \mathrm{~N} / \mathrm{cm}$. PET, PDO, and PLGA1090 meshes did not meet one or more of the threshold values, and therefore are not suitable candidates for use in hernia repair applications. Although PU met or surpassed all of the threshold values, it is also not a viable candidate for hernia repair. During each test, the
PU mesh underwent large amounts of elastic deformation before it reached its peak load. This is demonstrated by the $>1,100 \%$ elongation of the PU mesh during UTS testing. If PU mesh was implanted over a hernia site, it would likely allow the wound to reopen and may even herniate through the wound along with the viscera before reaching a sufficient strength to stop the hernia site from opening any further.

PCL, PLGA8218, and PLLA met or surpassed all of the mechanical test threshold values. PCL had the highest TTS, and was the only material of the three to surpass both the 
threshold value and the MotifMESHTM value. The BBS and UTS of PCL, however, were lower than those of PLLA and PLGA8218. PLGA8218 had the highest UTS of the three materials, and was the only material tested with a SRS comparable to that of MotifMESHTM. The BBS of PLGA8218, however, was significantly lower than that of PLLA, and the TTS of PLG8218 was the lowest of the three. PLLA mesh had the highest BBS, as well as the second highest TTS and UTS of the three. The only test in which PLLA was outperformed by both PCL and PLGA8218 was SRS; however the SRS of PLLA was not significantly different than the 20 N SRS threshold value. Furthermore, the SRS testing protocol tests the force necessary to pull out a single suture. Assuming multiple sutures will be used to fix the mesh in place in vivo, the actual SRS of the implanted mesh would be many times that of the test value. PLLA also performed well compared to MotifMESHTM. Although MotifMESHTM outperformed PLLA in UTS and SRS testing, PLLA surpassed MotifMESHTM in BBS as well as TTS The RS of PLLA was also comparable to that of MotifMESHTM. Mesh stiffness is an important factor to consider because a mesh that is too stiff may restrict the patient's range of motion in the abdominal region, causing discomfort, while a mesh that is too flexible may be difficult for surgeons to handle during the hernia repair procedure [1]. Although no universal radial stiffness standards or testing procedures have been established by the medical community at this time, the fact that the stiffness of PLLA is nearly equal to that of MotifMESHTM, a commercial surgical mesh approved by the FDA, is encouraging. Therefore, we believe the electrospun PLLA mesh is the most suitable material for use in hernia repair applications.

PLLA also offers several advantages from an in vivo degradation standpoint. PCL, PDO, PLGA1090, PLGA8218, and PLLA are all bioresorbable polymers, and therefore break down by natural processes in vivo. One advantage of resorbable meshes is a reduction of the long term foreign body response that often persists in patients with permanent mesh implants [16]. Due to chemical and structural differences, all of these polymers degrade at different rates. PDO and PLGA1090, for example, are completely resorbed in a matter of weeks post implantation. This short degradation profile does not give the hernia site adequate time to heal before the supporting mesh is gone, which may result in a high rate of recurrence in the years following a procedure [17]. PLLA, however, degrades over the course of 9-12 months. In some circumstances, this may provide enough time for native fascia to grow and remodel at the hernia site before the mechanical load is transferred from the mesh to the healed tissue. For example, a recent pilot study on the use of a resorbable mesh plug for inguinal hernia repair came to the conclusion that the plug was suitable for use in young and healthy patients with minor hernias [18].

While fully resorbable meshes may be useful in some circumstances, they will likely never be suitable for general use in hernia repairs. There is a growing body of evidence which suggests a genetic predisposition to hernia formation and recurrence in some patients $[19,20]$. A large portion of patients with recurrent hernias have a genomic profile displaying decreased expression of type I collagen and increased expression of type III collagen. This compromises the mechanical integrity of the patient's connective tissue, and increases the likelihood of hernia formation and recurrence. In cases such as these, even if a resorbable mesh led to the complete regrowth and remodeling of healthy fascia, patients would still be prone to recurrences after the mesh was resorbed.

A composite mesh made up of an electrospun PLLA layer backed by a permanent mesh could be a solution to this issue. The electrospun PLLA mesh would adhere to the abdominal wall and promote tissue growth across the defect during the initial stages of wound healing. As the PLLA layer gradually degrades in the months following implantation, it would transfer the mechanical load onto the newly formed fascia and the permanent mesh layer. The permanent mesh would remain and play a permanent, supplemental role in order to prevent recurrences. Furthermore, this permanent mesh layer could have large pores and a low mesh density to reduce chronic foreign body reaction. This mesh could be produced by electrospinning a nanofiber layer directly on to a polypropylene sheet coated with a thin layer of resorbable adhesive to prevent delamination.

One study suggests that composite materials result in an acute inflammatory response as the resorbable polymer degrades, usually into acidic byproducts, and offer no benefits over simple permanent meshes [16]. The composites tested in this study were composed of a permanent mesh fixed to a resorbable film, and films may not offer any significant benefits to counteract the negative impact of local inflammation in response to their degradation. Furthermore, these films are often used as adhesion barrier layers and have no direct interaction with the hernia site [5]. Conversely, it has been demonstrated that resorbable nanofiber layers in direct contact with the hernia site promote collagen deposition and remodeling, and yield better mechanical properties at the wound interface compared to polypropylene mesh alone [21]. This suggests that if the adhesion barrier layer was made from nanofibers instead of a film, there would be a significantly lower inflammatory response.

\section{Conclusions}

Electrospun nanofiber scaffolds have the potential to provide a cheap, reproducible hernia mesh with customized mechanical properties, degradation rates and an excellent capacity to support native tissue regeneration, but to date there has been no published literature characterizing electrospun meshes for hernia repair. In this work, novel electrospun nanofiber meshes were successfully manufactured and characterized using standardized tests for hernia meshes from ISO and ASTM in order to set a universal foundation those others can use in the future development of medical devices. The data demonstrates that PCL, PLGA8218, and PLLA electrospun meshes met or exceeded the minimum mechanical requirements for use in hernia repair applications. The mechanical properties of electrospun PLLA mesh make it an ideal candidate for further development. Future studies will need to be performed to evaluate the in vivo response of these electrospun nanofiber meshes prior to clinical translation. 


\section{References}

1. Bringman S, Conze J,Cuccurullo D, Deprest J, Junge K, Klosterhalfen B, et al. Hernia repair: the search for ideal meshes. Hernia. 2010; 14(1): 81-87. doi: 10.1007/s10029-009-0587-x.

2. Deeken CR, Faucher KM, Matthews BD. A review of the composition, characteristics, and effectiveness of barrier mesh prostheses utilized for laparoscopic ventral hernia repair. Surg Endosc. 2012; 26(2):56675. doi: 10.1007/s00464-011-1899-3.

3. Pott PP, Schwarz ML, Gundling R, Nowak K, Hohenberger P, Roessner ED. Mechanical Properties of Mesh Materials Used for Hernia PLoS One. 2012;7(10):e46978. doi: 10.1371/journal.pone.0046978.

4. Deeken CR, Abdo MS, Frisella MM, Matthews BD. Physicomechanical evaluation of absorbable and nonabsorbable barrier composite meshes for laparoscopic ventral hernia repair. Surg Endosc. 2011; 25(5):1541-52. doi: 10.1007/s00464-010-1432-0.

5. Jenkins ED, Yom V, Melman L, Brunt LM, Eagon JC, Frisella MM, et al Prospective evaluation of adhesion characteristics to intraperitoneal mesh and adhesiolysis-related complications during laparoscopic re-exploration after prior ventral hernia repair. Surg Endosc. 2010; 24(12):3002-7. doi: 10.1007/s00464-010-1076-0.

6. Rosen MJ. Biologic Mesh for Abdominal Wall Reconstruction: A Critical Appraisal. Am Surg. 2010; 76(1):1-6.

7. Candage R, Jones K, Luchette FA, Sinacore JM, Vandevender D, Reed RL 2nd. Use of human acellular dermal matrix for hernia repair: Friend or foe? Surgery. 2008; 144(4):703-9; discussion 709-11. doi: 10.1016/j. surg.2008.06.018.

8. Kim HS, Yoo HS. Therapeutic application of electrospun nanofibrous meshes. Nanomedicine (Lond). 2014; 9(4):517-33. doi: 10.2217/ nnm.13.224.

9. Johnson J, Ghosh A, Lannutti J. Microstructure-property relationships in a tissue-engineering scaffold. Journal of Applied Polymer Science, 2007; 104(5): 2919-2927. DOI: 10.1002/app.25965.

10. Johnson J, Niehaus A, Nichols S, Lee D, Koepsel J, Anderson D, et al. Electrospun PCL in Vitro: a Microstructural Basis for Mechanical Property Changes. J Biomater Sci Polym Ed. 2009; 20(4): 467-81. doi: $10.1163 / 156856209 \times 416485$.

11.Johnson J, Nowicki MO, Lee CH, Chiocca EA, Viapiano MS, Lawler SE, et al. Quantitative Analysis of Complex Glioma Cell Migration on Electrospun Polycaprolactone Using Time-Lapse Microscopy. Tissue Eng Part C Methods. 2009 Dec;15(4):531-40. doi: 10.1089/ten. TEC.2008.0486.

12. Agudelo-Garcia PA, De Jesus JK, Williams SP, Nowicki MO, Chiocca EN,
Liyanarachchi S, et al. Glioma Cell Migration on Three-dimensional Nanofiber Scaffolds Is Regulated by Substrate Topography and Abolished by Inhibition of STAT3 Signaling. Neoplasia. 2011 Sep; 13(9): 831-840.

13. Ebersole GC, Buettmann EG, MacEwan MR, Tang ME, Frisella MM, Matthews BD, et al. Development of novel electrospun absorbable polycaprolactone (PCL) scaffolds for hernia repair applications. Surg Endosc. 2012; 26(10): 2717-28.

14. Deeken CR, Abdo MS, Frisella MM, Matthews BD. Physicomechanical Evaluation of Polypropylene, Polyester, and Polytetrafluoroethylene Meshes for Inguinal Hernia Repair. J Am Coll Surg. 2011; 212(1): 6879. doi: 10.1016/j.jamcollsurg.2010.09.012.

15. Biomedical P. MotifMESH: MacroPorous NW Implant. 2008.

16. Sandor M, Scott N, Edwards M, Qi S, De Deyne P. In vitro and in vivo characterization of a fully resorbable and composite surgical mesh. Journal of Bioactive and Compatible Polymers. 2014; 29(2): 121-136. doi: 10.1177/0883911513520382.

17. Symeonidis D, Efthimiou M, Koukoulis G, Athanasiou E, Mamaloudis I, Tzovaras G. Open inguinal hernia repair with the use of polyglycolic acid/trimethylene carbonate absorbable mesh: a critical update of the long-term results. Hernia. 2013; 17(1): 85-7. doi: 10.1007/s10029012-1016-0.

18. Negro P, Campanelli G, Ipponi PL, Gossetti F, Dassatti MR, Manto O, et al. Selective use of bioabsorbable Gore BIO-A plug and patch for groin hernia repair : comment to: open inguinal hernia repair with the use of polyglycolic acid/trimethylene carbonate absorbable mesh: a critical update of the long term results. Symeonidis D, Efthimiou M, Koukoulis G, Athanasiou E, Mamaloudis I, Tzovaras G. Hernia 2013, 17:85-87. Hernia. 2013; 17(6): 795-6. doi: 10.1007/s10029-013-1117-4.

19. Calaluce R, Davis JW, Bachman SL, Gubin MM, Brown JA, Magee JD, et al. Incisional hernia recurrence through genomic profiling: a pilot study. Hernia. 2013; 17(2): 193-202. doi: 10.1007/s10029-012-09234.

20. Klinge U, Zheng H, Si Z, Schumpelick V, Bhardwaj RS, Muys L, et al. Expression of the extracellular matrix proteins collagen I, collagen III and fibronectin and matrix metalloproteinase- 1 and-13 in the skin of patients with inguinal hernia. Eur Surg Res. 1999; 31(6): 480-90.

21.Plencner M, East B, Tonar Z, Otáhal M, Prosecká E, Rampichová M, et al. Abdominal closure reinforcement by using polypropylene mesh functionalized with poly-epsilon-caprolactone nanofibers and growth factors for prevention of incisional hernia formation. Int J Nanomedicine. 2014; 9: 3263-77. doi: 10.2147/IJN.S63095. 